\title{
A Case Report of a Cecal Bascule with Internal Herniation through the Foramen of Winslow
}

\author{
Eric Mulkey ${ }^{1}$, Gregory Stewart ${ }^{1}$, Ernesto Enrique ${ }^{1}$, and Rafik El-Sabrout ${ }^{1}$ \\ ${ }^{1}$ Garnet Health Medical Center
}

October 20, 2021

\begin{abstract}
Internal hernias are a rare phenomenon, and even rarer is a herniation through the foramen of Winslow. We report a rare case of an 81 year old female presenting with vague abdominal symptoms who was found to have a cecal bascule herniating through the foramen of Winslow treated with surgery.
\end{abstract}

\section{Introduction:}

Internal hernias are a protrusion of a viscus through a normal or abnormal peritoneal or mesenteric aperture within the confines of the peritoneal cavity $(1,2,3)$. Such orifices can either be acquired, such as a postsurgical or post-traumatic defect, or congenital, including both normal anatomic apertures, such as the foramen of Winslow, and abnormal apertures arising from anomalies of internal rotation and peritoneal attachment $(1,3)$. Internal hernias account for approximately 0.5 to $6 \%$ of all cases of intestinal obstruction and can be associated with a mortality rate as high as $50 \%$ if gone undiagnosed or if there is delay in treatment $(1,2)$. Also, reports have shown that internal hernias are found on almost $1 \%$ of autopsies $(2)$. There are multiple different types of internal hernias that have been discussed in literature, and herniation through the foramen of Winslow is one of the less common subtypes. Furthermore, different organs can internally herniate through apertures, such as small bowel, appendix, and colon to name a few. The diagnosis of an internal hernia can be difficult to make base on clinical presentation as the patient can only complain of non-specific abdominal complaints. Patients can have radiologic findings of distended bowel, bowel wall thickening, mesenteric edema, and ascites. Surgical exploration based on presentation, physical examination, radiographic and laboratory findings is often necessary to make the diagnosis of internal hernia. Exploration allows for a therapeutic intervention to resolve the issue and/or prevent a recurrence. What follows is a rare case presentation and discussion of an elderly female presenting with vague abdominal symptoms who was taken to the operating room and found to have a cecal bascule herniating through the foramen of Winslow.

\section{Case Presentation}

An 81 year old female with a past medical history of microscopic colitis being treated with chronic prednisone (per patient report, she was diagnosed after colonoscopy at an outside facility multiple years ago) and wellcontrolled asthma and no past surgical history originally presented to an urgent care for the complaint of abdominal pain. She stated that she developed abdominal distention two to three months ago that she attributed to "old age" and now developed some cramping abdominal pain. There were no complaints of nausea or vomiting. She was passing flatus but endorsed recent constipation. At urgent care, a CT abdomen pelvis with IV and PO contrast showed concerns for a cecal volvulus and fecal impaction within the colon (Figure 1 and 2). She was sent to the emergency department for further evaluation and treatment.

Upon arrival to the ED, the patient was afebrile and hemodynamically stable. Her abdominal exam revealed an abdomen that was softly distended with mild upper abdominal tenderness. There were no peritoneal signs. 
Her laboratory work showed a leukocytosis of $12 \times 10^{9} / \mathrm{L}$, otherwise her CBC and CMP were unremarkable. The patient had a chest $\mathrm{x}$-ray and an abdominal x-ray in the emergency department that showed colonic distention but no pneumoperitoneum (Figure 3).

The patient was admitted to the hospital and taken to the operating room for an exploratory laparotomy. Intra-operatively, the small bowel was found to be non-dilated and healthy. However, the cecum was not found in its normal anatomic location. Further exploration revealed a markedly dilated colon from the transverse colon to the sigmoid. Ongoing exploration revealed a distended structure traversing into the lesser sac and posterior to the stomach. The gastro-colic ligament was divided along the greater curve of the stomach to gain access to the lesser sac. This revealed the presence of the appendix and a dilated cecum and part of the ascending colon (Figure 4). The cecum had herniated through the foramen of Winslow.

At this point, the decision was made to perform a right hemicolectomy. The transverse colon was mobilized and transected at the mid-colon using a GIA stapler. Attempt at manual reduction of the herniated bowel did not succeed due to the massively dilated cecum, which contained semi-solid stool. A purse-string suture was placed and a cecotomy was performed for decompression with minimal spillage. This allowed the cecum and ascending colon to be adequately reduced from its internal herniation. Upon reduction, it had appeared that the cecum had folded anteriorly over the ascending colon.

The cecum and ascending colon was then mobilized along the white line of Toldt, and a GIA stapler was used to transect the terminal ileum. Complete mobilization of the right colon and transverse colon was made by dividing the mesentery with a Ligasure. The specimen was removed and sent for pathological examination. An uncomplicated stapled anastomosis was then created between the terminal ileum and the remainder of the transverse colon in a side-to-side functional end-to-end fashion. Hemostasis was assured, the abdomen was irrigated with warm saline, and the abdomen was then closed. There were no intra-operative complications, and the patient was extubated postoperatively and returned to the medial/surgical floor in stable condition.

The patient's postoperative course was complicated by a prolonged ileus that eventually resolved with supportive care. On postoperative day 11, the patient was discharged after having adequate bowel function and tolerating a regular diet. Pathology revealed focal mural hemorrhage and mucosa with ischemic-type change of the colon consistent with an incarcerated hernia. The margins of resection were viable. The appendix and small bowel were unremarkable. The patient was seen in surgical clinic 2 weeks postoperatively and has had no issues postoperatively.

\section{Discussion:}

Internal hernias occur when there is a protrusion of a viscera through the peritoneum or mesentery and confined within the abdominal cavity $(1,2,3)$, and they are an uncommon source of intestinal obstructions. The rate of an internal hernia being the cause of an intestinal obstruction ranges from 0.5 to $4 \%$ of cases (2). Internal hernias are classified according to location and include paraduodenal (50-55\%), pericecal (10-15\%), foramen of Winslow (8\%), transmesenteric and transmesocolic (8\%), pelvic and paravesical (6\%), sigmoid $(6 \%)$, and transomental $(1-4 \%)(1,2,3,4,6)$

Hernias through the foramen of Winslow are a rare subset and was what our patient was found to have intra-operatively. The foramen of Winslow, or the epiploic or omental foramen, is a normal anatomical orifice that allows connection between the greater and lesser peritoneal cavities. Its anatomical borders are as follows: the free border of the hepatoduodenal ligament anteriorly, the inferior vena cava covered by the peritoneum posteriorly, the caudate lobe of the liver superiorly, and the first portion of the duodenum and the hepatic artery inferiorly. Under normal circumstances, the foramen of Winslow remains closed due to the intra-abdominal pressure. However, a hernia through the foramen can occur and accounts for up to $8 \%$ of internal hernias and $0.08 \%$ of all hernias $(1,2,3,4,5,6,8)$.

Risk factors for hernias via the foramen of Winslow include an enlarged foramen (the opening is normally approximately $3 \mathrm{~cm}$ ), an abnormally long small-bowel mesentery, persistence of the ascending mesocolon allowing for an excessively mobile bowel, an elongated right hepatic lobe which is thought to direct the 
mobile intestinal loops toward the foramen of Winslow, and changes in intra-abdominal pressures $(1,2,3,4$, 8 ). Other reported predisposing factors are the presence of an abnormally long and redundant bowel, history of a cholecystectomy, a "wandering cecum," and defects of the gastro-hepatic ligaments (8).

Foramen of Winslow hernias most commonly contain small bowel alone. However, about one-third of these hernias contain cecum and ascending colon, as in our patient $(1,2,3,4,6,8)$. Even rarer is an internal hernia involving the transverse colon, omentum, and/or gallbladder.

Internal hernias often have vague, non-specific clinical presentations making the diagnosis difficult before pursuing adjuncts for additional information. Patients range from being asymptomatic to significant abdominal pain with peritonitis. Common complaints include obstructive symptoms, such as nausea, vomiting, anorexia, decreased bowel function, abdominal bloating/distention, and/or cramping abdominal pain. These symptoms may be triggered by changes in intra-abdominal pressure, such as pregnancy, straining, or during the postprandial period, and are sometimes relieved by sitting forward $(1,8)$. On clinical exam, the abdomen is often distended with associated tenderness frequently in the upper abdomen. The typical patient presenting with an internal hernia through the foramen of Winslow is a middle-aged person with acute onset of pain that becomes progressive and associated with obstructive signs and symptoms $(1,2,8)$.

Due to a non-specific clinical presentation, further workup with radiologic imaging is often necessary. A computed tomography (CT) scan of the abdomen and pelvis is the gold-standard diagnostic imaging study. Abdominal X-ray is neither sensitive nor specific for a foramen of Winslow hernia, but can be used to add more clinical information. Characteristic plain film radiographic findings include gas-containing intestinal loops in the left upper abdomen medial to the lesser curvature of the stomach causing displacement of the stomach anteriorly and laterally $(2,8)$. Furthermore, the transverse colon may be displaced inferiorly by the hernia and lie underneath the abnormal collection of gas. If the colon, particularly the cecum or ascending colon, is involved in the hernia, there will be no bowel gas or stool evident in the right iliac fossa (8).

CT scan of the abdomen and pelvis is the imaging modality of choice due to its availability, speed, and detailed reformatting capabilities. On CT, multiple gas-filled loops of bowel will be located in the lesser sac, posterior to the liver hilum, anterior to the IVC, and between the stomach and pancreas, with tapering of the herniation though the foramen of Winslow $(1,2,8)$. Other characteristic features include the presence of the mesentery between the IVC and portal vein, absence of the ascending colon in the right gutter, two or more bowel loops in the high subhepatic spaces, and/or displacement of the stomach anteriorly and laterally $(2,8)$. If the cecum is involved, it often appears as a cecal volvulus being present (1). If the terminal ileum is the herniated organ, a closed-loop obstruction often ensues behind the portal vein resulting in portal venous compression and narrowing, which could also be a useful sign to diagnose a hernia through the foramen of Winslow (8).

Even after imaging, the diagnosis of a foramen of Winslow hernia is only accomplished about $10 \%$ of the time pre-operatively (7). As mentioned above, a cecal volvulus is often a leading differential as it can appear similar on CT scan. What makes our case even more interesting is that our patient was found to have a cecal bascule, a type of cecal volvulus, in addition to a foramen of Winslow hernia.

A cecal bascule is a rare cause of bowel obstruction in which a mobile cecum folds anteriorly and superiorly over the ascending colon $(3,4,5)$. Cecal volvulus accounts for $1-2 \%$ of all large bowel obstructions, and $5-20 \%$ of those cases are a cecal bascule (5). A cecal bascule is different from a cecal volvulus as there is no axial rotation of the colon and no mesenteric vascular compromise, thus ischemia would only occur from intraluminal tension or extraluminal compression from the borders of the foramen of Winslow $(3,4,5)$. Due to the lower risk of vascular compromise, patients with a cecal bascule present with less critical illness than those with a cecal volvulus (4). A well-known "whirl sign" on CT scan is specific for a cecal volvulus with an axial torsion due to its mesenteric twisting, whereas a cecal bascule can present with signs such as displacement of the cecum to the upper or mid abdomen, displacement of the ileocecal valve to the right upper quadrant, a transition zone between the cecum and ascending colon, and/or perihepatic free fluid (5).

As mentioned above, the diagnosis of a foramen of Winslow internal hernia is often not confirmed until in 
the operating room, so surgical intervention is necessary to diagnose and treat the problem. The surgical management requires reduction in the presence of obstructive symptoms with care to evaluate the herniated bowel for non-reversible ischemia $(3,7)$. Surgical options after reduction of the internal hernia if the bowel is viable is a right hemicolectomy, an appendectomy with cecopexy, or hernia reduction with a cecostomy (6). A right hemicolectomy is often performed in order to limit the chance of a recurrence of both an internal hernia and cecal bascule or cecal volvulus $(6,7,8)$. However, there has been no consensus on standard operative intervention as this is a very rare surgical finding, but a right hemicolectomy is most often performed.

Laparotomy is often used rather than laparoscopy as patients often have dilated bowel, but some have advocated laparoscopy in select patients based on surgeon discretion. In terms of reducing the internal hernia, it is often accomplished via gentle traction. However, opening of the lesser sac, enlarging the foramen via a Kocher maneuver, and/or needle decompression may be needed to facilitate reduction if there is massive colonic dilatation $(7,8)$. Closure of the foramen of Winslow is often advocated against to prevent a recurrence due to the risk of portal vein thrombosis or damage to the portal triad $(4,8)$.

\section{Conclusion:}

Internal hernias through the foramen of Winslow are uncommon, and a concomitant cecal bascule involved in the herniation is even more of a rarity. Patient presentation with internal hernias are often non-specific and sometimes with obstructive signs and symptoms. Expediting radiologic imaging with a CT scan of the abdomen and pelvis is vital in the workup in order to prevent delaying a diagnosis and allowing potential progression to ischemic bowel. Surgical intervention is the mainstay treatment of a foramen of Winslow internal hernia and often involves a right hemicolectomy in order to limit the likelihood of a recurrence. Further research to determine a standard operative intervention and the role of laparoscopy in treatments is still necessary for the treatment of internal hernias through the foramen of Winslow.

Acknowledgements: A special thanks to Dr. Charles Fasanya, MBBS for leaving a lasting impact on our surgical residency program at Garnet Health Medical Center.

Consent: I confirm that written patient consent has been signed and collected in accordance with the journal's patient consent policy, and that I have added a patient consent statement asserting this at the bottom of the manuscript's title page. I will retain the original written consent form and provide it to the Publisher if requested.

\section{References:}

1. Martin LC, Merkle EM, Thompson WM. Review of internal hernias: radiographic and clinical findings. AJR Am J Roentgenol. 2006 Mar;186(3):703-17. doi: 10.2214/AJR.05.0644. PMID: 16498098.

2. Takeyama N, Gokan T, Ohgiya Y, Satoh S, Hashizume T, Hataya K, Kushiro H, Nakanishi M, Kusano M, Munechika H. CT of internal hernias. Radiographics. 2005 Jul-Aug;25(4):997-1015. doi: 10.1148/rg.254045035. PMID: 16009820.

3. Rajeswaran G, Selvakumar S, King C. Internal herniation of the caecum into the lesser sac: an unusual cause of an acute abdomen (2009: 10b). Eur Radiol. 2010 Jan;20(1):249-52. doi: 10.1007/s00330-0091450-2. Epub 2010 Jan 7. PMID: 20063081.

4. Makarawo T, Macedo FI, Jacobs MJ. Cecal bascule herniation into the lesser sac. World J Clin Cases. 2014 Dec 16;2(12):903-6. doi: 10.12998/wjcc.v2.i12.903. PMID: 25516868; PMCID: PMC4266841.

5. Lung, B.E., Yelika, S.B., Murthy, A.S. et al. Cecal bascule: a systematic review of the literature. Tech Coloproctol 22, 75-80 (2018). https://doi.org/10.1007/s10151-017-1725-6

6. Ayoob F, Michael R, Chadnick Z, Fasanya C. Internal hernia of caecum through the foramen of Winslow. BMJ Case Rep. 2019 Jul 1;12(7):e228239. doi: 10.1136/bcr-2018-228239. PMID: 31266756; PMCID: PMC6605905.

7. Downs P, Downes N, Zayshlyy E, Esper C, Giuseppucci P. Internal hernia through the foramen of Winslow. J Surg Case Rep. 2018 Dec 11;2018(12):rjy329. doi: 10.1093/jscr/rjy329. PMID: 30555674; PMCID: PMC6289221. 
8. Moris D, Tsilimigras DI, Yerokun B, Seymour KA, Guerron AD, Fong PA, Spartalis E, Sudan R. Foramen of Winslow Hernia: a Review of the Literature Highlighting the Role of Laparoscopy. J Gastrointest Surg. 2019 Oct;23(10):2093-2099. doi: 10.1007/s11605-019-04353-3. Epub 2019 Aug 16. PMID: 31420858.

Figures and Images:

Figure 1 - Axial CT scan showing the colon herniating through the Foramen of Winslow and posterior to the stomach

Figure 2 - Coronal CT scan showed bowel herniation through the Foramen of Winslow with dilated, stoolfilled colon

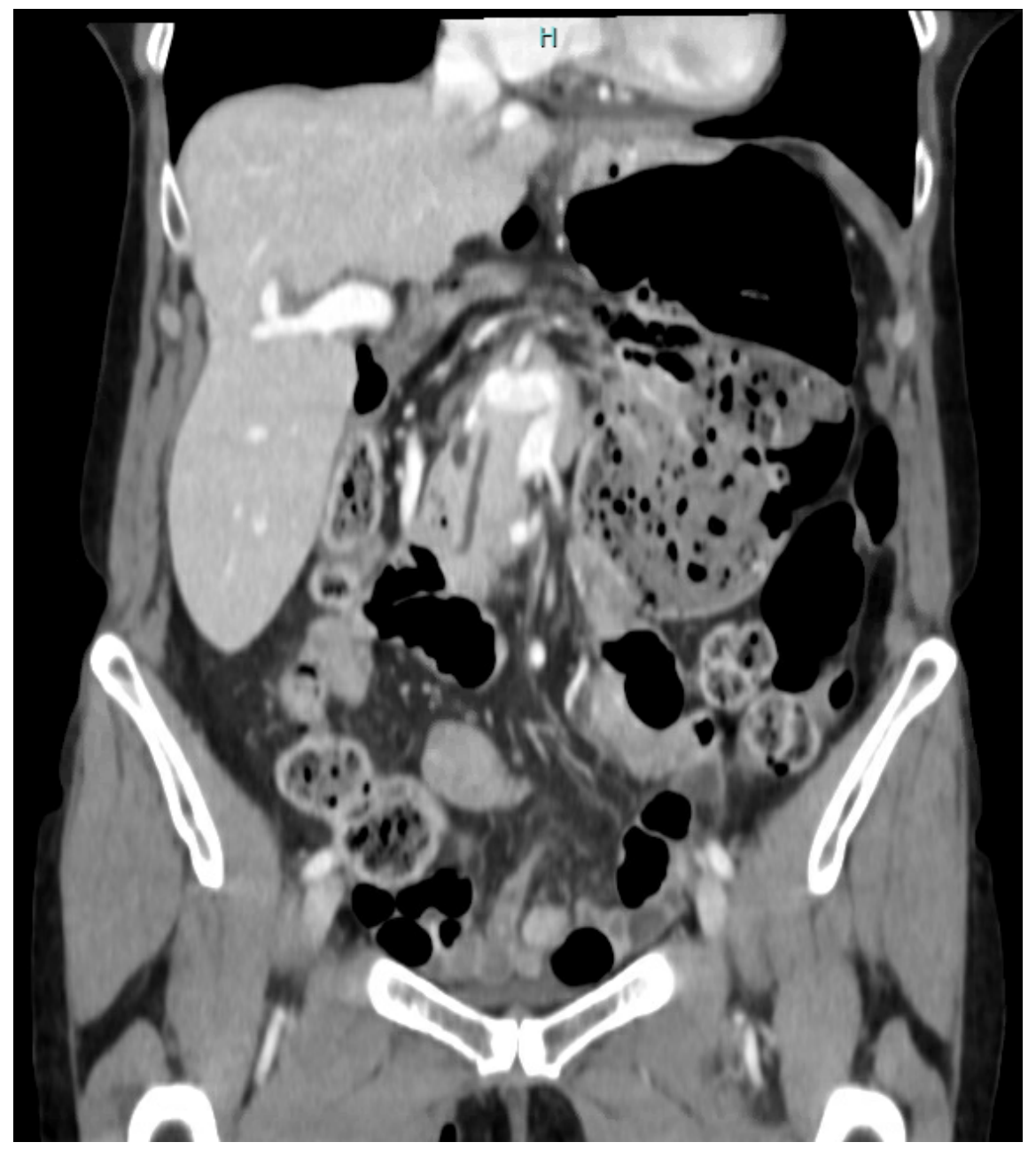

Figure 3 - Abdominal Xray with diffuse colonic dilation with a moderate amount of retained fecal matter 


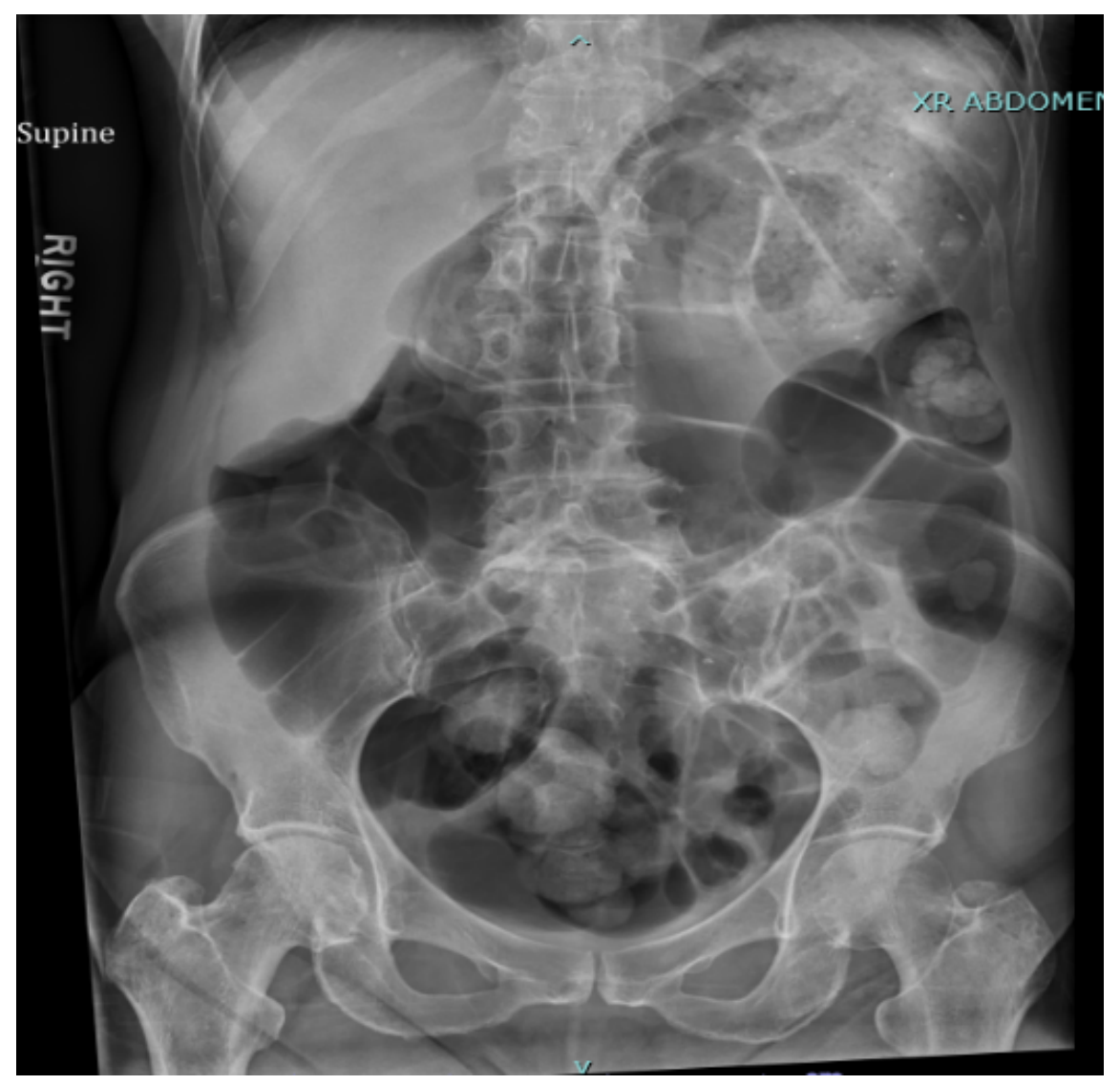

Figure 4 - Intra-operative picture of appendix and dilated cecum/ascending colon visualized posterior to the stomach once the lesser sac was entered

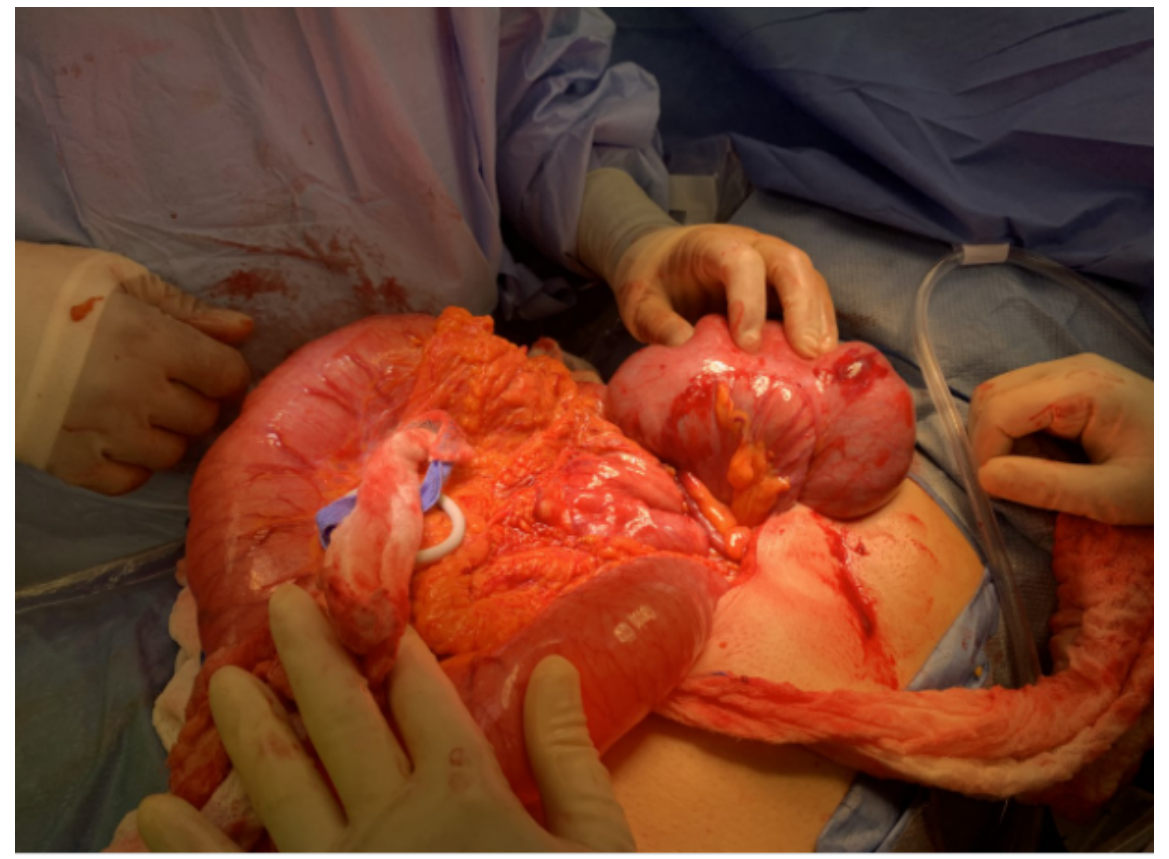

